\title{
Antigenic fingerprinting of diphtheria toxoid adsorbed to aluminium phosphate
}

\author{
Janny Westdijk ${ }^{\text {a, }}{ }^{*}$, Bernard Metz ${ }^{\text {a }}$, Nanda Spruit ${ }^{\text {a }}$, Wichard Tilstra ${ }^{\text {a }}$, Johan van der Gun ${ }^{\text {b }}$, \\ Coenraad Hendriksen ${ }^{\text {a, c }}$, Gideon Kersten ${ }^{\text {a, d }}$ \\ a Institute for Translational Vaccinology, P.O. Box 450, 3720 AL Bilthoven, The Netherlands \\ ${ }^{\mathrm{b}}$ Bilthoven Biologicals, P.O. Box 457, 3720 AL Bilthoven, The Netherlands \\ ${ }^{\mathrm{c}}$ Utrecht University, Department of Animals in Science and Society-3R Centre Utrecht Life Sciences, Yalelaan 2, P.0. Box 80.166, 3508TD Utrecht, The \\ Netherlands \\ ${ }^{\mathrm{d}}$ Leiden Academic Center for Drug Research, Drug Delivery Technology, P.O. Box 9502, 2300 RA Leiden, The Netherlands
}

\section{A R T I C L E I N F O}

\section{Article history:}

Received 14 April 2016

Received in revised form 10 October 2016

Accepted 17 October 2016

Available online 1 March 2017

\section{Keywords:}

Diphtheria toxoid

Aluminium phosphate

Adsorption

ELISA

Consistency

Antigenic fingerprinting

\begin{abstract}
A B S T R A C T
The antigenicity of alum-adsorbed diphtheria toxoid (DTd) was determined in combination vaccines, containing DTd, tetanus toxoid and inactivated poliovirus. A panel of monoclonal antibodies was used, covering five epitopes, distributed over the antigen. The resulting antigenic fingerprint of DTd demonstrates consistency of adsorption at antigen level in final product combination vaccines. The antigenic quality of DTd alone, adsorbed to aluminium phosphate, was also determined and compared with preadsorbed toxoid (starting material as well as toxoid desorbed from aluminium phosphate). Some epitopes became less accessible after adsorption, while others became relatively better exposed. Some epitopes disappeared almost completely upon adsorption, but were re-established after desorption of the antigen. The results indicate that DTd is adsorbed to aluminium phosphate in a preferred orientation and not randomly.
\end{abstract}

() 2016 International Alliance for Biological Standardization. Published by Elsevier Ltd. All rights reserved.

\section{Introduction}

Aluminium phosphate and aluminium hydroxide are commonly used adjuvants in many licensed human vaccines, e.g. vaccines containing diphtheria toxoid (DTd), tetanus toxoid (TTd) and inactivated poliovirus (IPV). Despite its widespread use for many decades, the mechanism of action of aluminium salts is not yet unraveled completely. Probably, several aspects contribute to the adjuvant effect of aluminium salts. Aluminium adjuvants can stimulate the immune system by inducing endogenous danger signals [1,2]. Aluminium salts may also act as an antigen delivery system forming a depot at the injection site [3,4], although this is questioned [5]. Small antigen-coated aluminium salt particles may deliver the antigen to antigen-presenting cells more efficiently as compared to soluble antigen [6]. Furthermore, it is likely that adsorption of antigen to aluminium salts contributes positively to the adjuvant effect. There may be an optimum in the binding

\footnotetext{
* Corresponding author.

E-mail address: janny.westdijk@intravacc.nl (J. Westdijk).
}

strength and the conformational stability of the antigen may play a role, but the degree of adsorption is considered important for induction of antibodies. Chang and co-workers obtained indications that the antibody response correlates with the degree of adsorption as it is in interstitial fluid [7]. This positive contribution of adsorption or adsorption strength on the antibody response was confirmed by others $[8,9]$. However, there is not always a positive effect of adsorption on the induction of antibodies [10,11]. Too strong binding may result in decreased immunogenicity [12]. Although the importance of antigen-adjuvant interaction may be antigen and species dependent, in general vaccine developers and manufacturers want to achieve high adsorption degrees and regulatory authorities demand consistent and stable adsorption process [13].

The turbidity of aluminium salts hampers quality control of the vaccine using in vitro methods and analytical techniques. For routine quality control, the degree of adsorption is usually established indirectly by either measuring the pre-adsorbed antigen in the supernatant or by desorbing the antigen from the alum salt. Desorption can be done by phosphate ions in case of aluminium hydroxide-adsorbed antigens [14] or by citrate buffer in case of 
aluminium phosphate-adsorbed antigens [15]. In all cases, measurements are not directly done on the adsorbed product. It has been shown that adsorbed antigen may have a different conformation as compared to the pre-adsorbed starting material [16]. For DTd it was shown that substantial conformational changes occur upon adsorption to aluminium hydroxide [17]. To assess vaccine quality, like batch-to-batch consistency and stability, there is a need to measure antigen quality in the final product, i.e. in adsorbed condition. Analysis can be done by physico-chemical analyses, e.g. fluorescence spectroscopy, circular dichroism and scanning calorimetry [16]. Although very informative, these methods can only be used for single antigens adsorbed to aluminium salts. This approach is not suitable in combination vaccines, because the methods cannot distinguish between antigens.

It is possible to quantify adsorbed antigen by direct immunoassay [18] or FACS analysis [19]. The Direct Alhydrogel Formulation Immunoassay (DAFIA) is an ELISA in which the solid phase is provided by the aluminium hydroxide. A fluorescein-conjugated antimouse antibody is used for detection of the antigen specific antibody bound to the aluminium hydroxide adsorbed antigen. The method was developed for a malaria antigen and showed excellent accuracy and good sensitivity, irrespective of the monoclonal antibodies that were used. As an alternative, a direct protein assay, using o-phthalaldehyde was also available for aluminium-adsorbed antigens [20]. However, this method was less sensitive and of course not antigen specific. Alternatively, a FACS-based assay has been described using polyclonal rabbit anti-sera against meningococcal proteins [19]. As the detecting antibody, goat anti-rabbit Fab fragment was used labeled with a fluorophore (Alexa Fluor 647). An additional advantage of FACS analysis is that information on the particle size of aluminium hydroxide is obtained. For routine analysis, FACS may be less suitable since most quality control labs will not have access to the equipment.

In most vaccines, aluminium salt particles are not fully saturated with antigen and there is possibility for other proteins, e.g. blocking agent, monoclonal antibodies, conjugate, to adsorb during the assay to the aluminium salt, resulting in high background signals. On the other hand, the presence of antibodies during the assay may desorb part of the antigen. An equilibrium exists between the adsorption of all proteins involved: the antigen, the antibodies used for detection as well as the blocking agent that is needed to prevent non-specific adsorption of antibodies. In this paper, we use an adapted version of the DAFIA to perform a qualitative analysis of DTd adsorbed to aluminium phosphate, using a panel of antibodies. By comparing the antigenic fingerprints of different batches of DTdcontaining vaccines we investigate if product consistency can be demonstrated. In addition, we investigate whether epitope availability changes by adsorbing DTd to aluminum salts and whether epitope availability returns to pre-adsorption level after DTd desorption.

\section{Materials and methods}

\subsection{Antigens and vaccines}

DT-IPV vaccines were provided by Bilthoven Biologicals (BBio, The Netherlands). DT-IPV is a licensed product and all batches met release criteria. DT-IPV1-3 contained diphtheria toxoid bulk X (DTd-X), and DT-IPV4-6 contained bulk Y (DTd-Y). Also these preadsorbed diphtheria toxoids (DTd-X and DTd-Y) were obtained from BBio. Diphtheria toxin (DTx) was produced by Intravacc (The Netherlands).

The diphtheria toxin (DT79/1) is an in-house reference supplied by BBio containing $100 \mathrm{Lf} / \mathrm{ml}$ toxin. DT79/1 was used in the ELISA as a reference.

\subsection{Antibodies}

Anti-DTx monoclonal antibodies (mabs) Dim9, Dim24, Dim25 and Dim27 were produced at the National Institute of Public Health and the Environment (The Netherlands). All mabs recognize different epitopes located on A-fragment (Dim9 and Dim24) or Bfragment (Dim25, Dim27), as was determined by pair-wise binding of antibodies (primary antibody-antigen-second antibody) by Biosensor analysis [21]. Contrarily, Dim27 binds mainly to B-fragment of diphtheria toxin.

Dim9, Dim25 and Dim27 are toxin neutralising in the order Dim9 $=$ Dim25 $>$ Dim27. The epitope for Dim27 is largely destroyed in diphtheria toxoid by the formaldehyde treatment [22]. Furthermore, Dim9 inhibits the binding of mouse antiserum to diphtheria toxin, indicating that the epitope is immunodominant [21].

Anti-DTd monoclonal antibody DiD1 was purchased from Abnova (Taiwan) and binds to the B-fragment of DTx and DTd.

Horse anti-DTd serum (PaD) and horse anti-DTd peroxidase conjugate (PaDPO) were obtained from BBio.

HRPO-conjugated goat-anti-mouse IgG was bought from Southern Biotech (Birmingham, USA).

\subsection{Adsorption of DTd-X, DTd-Y and DTx to Adju-Phos}

Experimental vaccines adDTd-X, adDTd-Y and adDTx were prepared by adsorbing $15 \mathrm{Lf} / \mathrm{ml}$ of DTd-X, DTd-Y or DTx, respectively, to $0.33 \mathrm{mg} / \mathrm{ml} \mathrm{Al}^{3+}$ (Adju-Phos $2 \%$, Brenntag, Denmark) (Table 1$)$. The vaccines were incubated by stirring overnight $(\mathrm{o} / \mathrm{n})$ at $4{ }^{\circ} \mathrm{C}$, and then stored at $4{ }^{\circ} \mathrm{C}$ prior to the analysis.

\subsection{Desorption of DTd-X, DTd-Y and DTX}

Desorption of DTd or DTx from aluminium phosphate was achieved by adding $5 \%(\mathrm{w} / \mathrm{v})$ sodium citrate (Calbiochem, Merck Germany) and incubating the vaccines o/n at $37^{\circ} \mathrm{C}$.

\subsection{Sandwich ELISA for pre-adsorbed and desorbed DTd and DTx}

Polystyrene 96-well plates (Greiner Bio-one, Germany), were coated overnight at room temperature with $\mathrm{PaD}(0.6 \mathrm{AU} / \mathrm{ml})$ in $0.4 \mathrm{M}$ carbonate buffer, pH9.6. The plates were washed with tapwater containing $0.05 \%$ Tween 80 . DT79/1 and test samples were titrated using twofold dilutions series in buffer A (phosphatebuffered saline (PBS, pH 7.4), containing 0.5\% Protifar (Nutricia, the Netherlands) and 0.05\% Tween 80 (Merck, Germany), starting at 1.2 $\mathrm{Lf} / \mathrm{ml}$. Following incubation for $2 \mathrm{~h}$ at $37^{\circ} \mathrm{C}$, the plates were washed. Epitope-specific mabs or polyclonal antibodies were added (Dim9 1:8000; DiD1 1:4000; Dim24 1:1000, Dim25 and Dim27 1:2000; and PaDPO 1:2000 in buffer A) and the plates were incubated $1.5 \mathrm{~h}$ at $37{ }^{\circ} \mathrm{C}$. After washing the plates, HRPO-conjugated goat-antimouse IgG (1:2000 in buffer A; Southern Biotech) was added to each well, except for the wells containing PaDPO, followed by incubation at $37{ }^{\circ} \mathrm{C}$ for $2 \mathrm{~h}$. Plates were washed and tetramethylbenzidine (TMB) substrate (Sigma-Aldrich) was added to all wells. After $10 \mathrm{~min}$ the reaction was stopped by addition of $0.2 \mathrm{M} \mathrm{H}_{2} \mathrm{SO}_{4}$ and absorbance at $450 \mathrm{~nm}$ was measured. Antigen content was calculated relative to DT79/1 by parallel line analysis (log optical density vs. log dose), using a minimum of 3 sequential points from the linear section of the dose-response curve for the control and test sample.

\subsection{Immunoassay for aluminium-adsorbed DTd and DTx}

The immunoassay previously described by Zhu et al. [18] was used with some modifications. Test samples were added in 
Table 1

Vaccines used in this study.

\begin{tabular}{|c|c|c|c|c|}
\hline Vaccine & Manufacturer & Antigen & Antigen concentration (Lf/ml) & Conc $\mathrm{Al}^{3+}(\mathrm{mg} / \mathrm{ml})^{\mathrm{c}}$ \\
\hline DT-IPV1 & BBio $^{\mathrm{a}}$ & DTd-X & 2.5 & 0.33 \\
\hline DT-IPV2 & BBio & DTd-X & 2.5 & 0.33 \\
\hline DT-IPV3 & BBio & DTd-X & 2.5 & 0.33 \\
\hline DT-IPV4 & BBio & DTd-Y & 2.5 & 0.33 \\
\hline DT-IPV5 & BBio & DTd-Y & 2.5 & 0.33 \\
\hline DT-IPV6 & BBio & DTd-Y & 2.5 & 0.33 \\
\hline AdDTd-X & Intravacc $^{\mathrm{b}}$ & DTd-X & 15 & 0.33 \\
\hline AdDTd-Y & Intravacc & DTd-Y & 15 & 0.33 \\
\hline AdDTx & Intravacc & DTx & 15 & 0.33 \\
\hline
\end{tabular}

${ }^{a}$ Routinely produced vaccines by BBio (Bilthoven Biologicals).

b Experimental vaccines prepared by Intravacc.

c $\mathrm{Al}^{3+}$ in aluminium phosphate $2 \%$.

duplicate $(100 \mu \mathrm{l} /$ well) in twofold dilution series in buffer B (5\% fetal bovine serum (Hyclone, US) in $0.9 \% \mathrm{NaCl}$-solution) to black, 96-well V-bottom plates (Greiner Bio-One, Germany). As a negative control, a twofold dilution series of $\mathrm{AlPO}_{4}$ alone $\left(0.33 \mathrm{mg} / \mathrm{ml} \mathrm{Al}{ }^{3+}\right)$ was included. As a positive control a twofold dilution series of DTIPV6 with PaDPO (1:1000) as detecting antibody was added to each plate.

HRPO-conjugated goat-anti-mouse IgG (Southern Biotech)(1:1000) was mixed 1:1 with either Dim9 (1:4000), DiD1 (1:1000), Dim24 (1:250), Dim25 (1:250) or Dim27 (1:250) in buffer $\mathrm{B}$, and were added to the microplates $(100 \mu \mathrm{l} /$ well $)$ after preincubation for $30 \mathrm{~min}$ at $37{ }^{\circ} \mathrm{C}$. Otherwise, $100 \mu \mathrm{l} /$ well PaDPO alone $(1: 1000)$ in buffer $\mathrm{B}$ was added to the microtiter plates. The plates were shaken ( $300 \mathrm{rpm}$ ) at $37^{\circ} \mathrm{C}$ for $1 \mathrm{~h}$, followed by 5 washes with PBS and $0.05 \%$ Tween 80 by centrifugation at $2000 \mathrm{~g}$ for $4 \mathrm{~min}$ at room temperature. Next, following the final wash, pellets were resuspended in $100 \mu \mathrm{l}$ substrate consisting of equal volumes of Pico Stable Peroxide Solution (Pierce, USA) and Pico Luminol Enhancer Solution (Pierce, USA). The luminescence intensity was read by a microplate reader (Biotek Synergy MX, USA). The attribution to the luminescence intensity of the negative control $\left(\mathrm{AlPO}_{4}\right)$ at the 6th dilution was $<10 \%$ of the luminescence intensity of the positive control (DT-IPV6 with PaDPO). Moreover at this dilution, the luminescence intensity was measured for all mabs in case of DTIPV1-6 $(0.08 \mathrm{Lf} / \mathrm{ml})$ halfway the descending portion of the curve and in case of DTx $(0.5 \mathrm{Lf} / \mathrm{ml})$ in the beginning of the descending portion of the curve. Therefore, the binding intensity of mab or PaDPO was expressed as the luminescence intensity at the 6th dilution after subtraction of the luminescence intensity of the negative control at the 6 th dilution $\left(10 \mathrm{ng} / \mathrm{ml} \mathrm{Al}^{3+)}\right)$.

\subsection{Statistical analysis}

The two-way ANOVA with Tukey's multiple comparisons test was used for statistical analysis (GraphPad Prism 6.07 for Windows) (GraphPad Software, San Diego, USA). P values $<0.05$ were considered significant.

\section{Results}

\subsection{Quality of the immunoassay for aluminium-adsorbed DTd and} DTx

The immunoassay for aluminium-adsorbed DTd and DTx was performed with 6 DT-IPVs, 2 adDTds and 1 adDTx. The dose response curves obtained with DiD1 is given in Fig. 1. To check plate-to-plate variation, a positive control with PaDPO was included on each plate. Also, each plate contained a negative control (aluminium phosphate) to determine non-specific effects. The highest aluminium phosphate concentrations revealed some aspecific binding of mabs. The luminescence intensity of the negative control was subtracted from the luminescence intensity of the DTd or DTx in the same microplate (Fig. 1B and D). In order to be able to compare the results of DT-IPVs and DTd/DTx vaccines we kept the aluminium phosphate concentration of the different vaccines the same. The adsorbed experimental vaccines show a plateau in the response to DiD1 as compared to the DT-IPV vaccines, due to the higher DTd concentration (Fig. 1C and D). For the read-out of the results we chose the luminescence intensity at 64 times diluted samples. At that dilution the background was low and constant and the specific signal was easily measurable. With regard to the DT-IPV vaccines, the average luminescence intensity of the positive control after subtraction of the negative $\left(\mathrm{AlPO}_{4}\right)$ control at the 64 times dilution was $4.2 \mathrm{E} 5$ with a variation coefficient of $7.3 \%(n=14)$. Regarding adDTd-X, adDTd-Y and adDTX vaccines the average luminescence intensity was $4.6 \mathrm{E} 5$ with a variation coefficient of $14.2 \%(\mathrm{n}=14)$.

\subsection{Antigenic fingerprints of DT-IPV vaccines}

Six final vaccine lots, DT-IPV1 - DT-IPV6, were analysed in the aluminium-adsorbed antigen immunoassay against a panel of monoclonal antibodies (Dim9, Dim24, Dim25, Dim27 and DiD1) and polyclonal PADPO. Antigenic fingerprints were prepared of DTIPV1 - DT-IPV6 by measuring the binding intensities of the mabs and PadPO (Fig. 2A).

No significant differences were detected between DT-IPV1 - DTIPV6 using Dim9, Dim27 or the polyclonal antibody PaDPO $(p>0.05)$. Dim 24 had a significant lower binding to DT-IPV4 as compared to the other batches. Dim25 and DiD1 had a significantly higher binding intensity to DT-IPV5 than to other batches. With Dim25, DT-IPV5 was significantly higher than DT-IPV1 and DT-IPV2 $(\mathrm{p}=0.02)$. With DiD1, DT-IPV5 was higher than DT-IPV1 and DTIPV3 ( $p<0.01$ ). Dim27 showed hardly any binding to DT-IPV1 DT-IPV6, indicating that in adsorbed state the epitope for Dim27 was either shielded by aluminium phosphate or had disappeared by conformational changes.

Furthermore, Fig. 2B shows the antigenicity of the DTd in DT-IPV vaccines to a panel of mabs and PaDPO after desorption from aluminium phosphate.

After desorption, differences in antigen binding ( $\mathrm{Lf} / \mathrm{ml}$ ) between DT-IPV1 - DT-IPV6 became more prominent than in adsorbed state. From all DT-IPV vaccines, DT-IPV1 had the lowest binding intensities which was significant for Dim24, DiD1 and PadPO ( $p<0.002)$. DT-IPV5 showed the highest antigenicity (Fig. 2B) and differed significantly with the other five DT-IPV vaccines when using Dim24 and DiD1 ( $<<0.004)$. DT-IPV2, DTIPV3, DT-IPV4 and DT-IPV6 were equally antigenic with mabs 
A

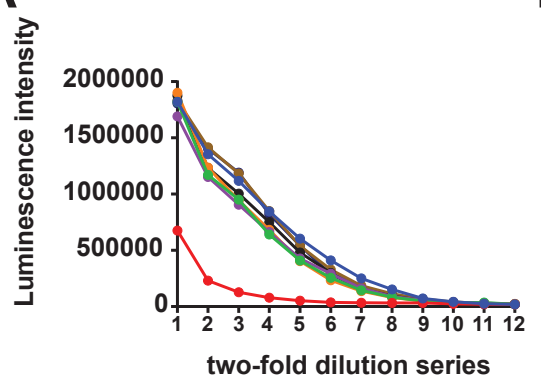

$\rightarrow$ positive control $\quad \rightarrow$ DT-IPV1
$\rightarrow$ negative control

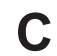

B

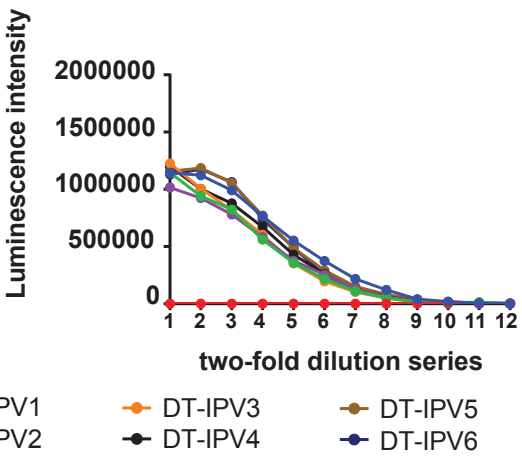

童

C

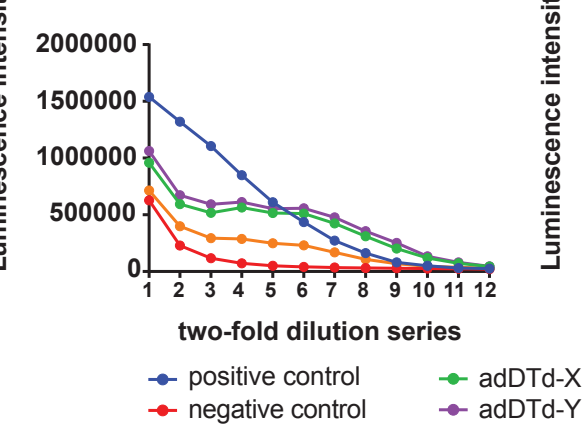

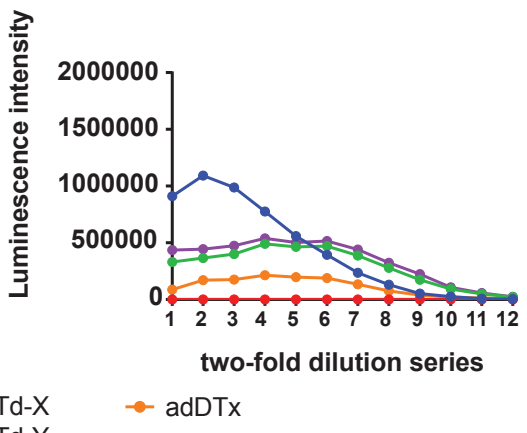

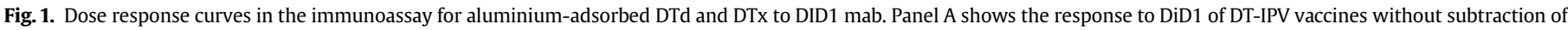

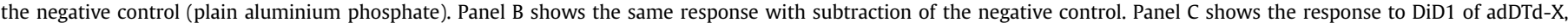
adDTd-Y and adDTx without subtraction of the negative control, Panel D shows the same response as in panel $\mathrm{C}$ with subtraction of the negative control.

Dim9, Dim24, Dim25 and Dim27 ( $p>0.05$ ).

Although in adsorbed state no binding of Dim27 was observed to DT-IPV1 - DT-IPV6 (Fig. 2A), Dim27 showed considerable binding to DTd after desorption from DT-IPV1 - DT-IPV6 (Fig. 2B).

\subsection{Antigenic fingerprints of adsorbed and desorbed DTd-X and DTd-Y}

DTd-X and DTd-Y used for the production of DT-IPV vaccines, were also applied to examine the effect of adsorption and desorption on the antigenic fingerprints. All antibodies showed that DTd$X$ and DTd-Y were antigenically rather identical (Fig. 3B). Before adsorption the differences between DTd-X and DTd-Y were not significantly different, except for DiD1 ( $p=0.0005)$ (Fig. 3B). Contrarily, desorbed DTd-X was significantly higher than DTd-Y regarding to $\operatorname{Dim} 27$ ( $p=0.01$ ). Unexpectedly, the antigenic concentrations of DTd-X and DTd-Y were slightly higher after desorption (desorbed state) than before adsorption (pre-adsorbed state). The differences in concentrations pre-adsorption and postdesorption were significant for Dim25, (DTd-Y, $p=0.007$ ), Dim27 (DTd-X, p $<0.0001$ ) and DiD1 (DTd-X and DTd-Y, p < 0.0009) (Fig. 3B).

Also, the antigenic fingerprints of experimentally adsorbed toxoids adDTd-X and adDTd-Y were not significantly different ( $p>005$ ) (Fig. 3A), demonstrating consistent adsorption step. The epitope for Dim27 was almost absent in adDTd-X and adDTd-Y, as for the commercial DT-IPVs, but the epitope was detectable in preadsorbed and desorbed DTd-X and DTd-Y (Fig. 3B). In adsorbed state the binding intensities of Dim24, Dim25, DiD1 and PaDPO were much higher than of Dim9, and Dim27 (Fig. 3A). These results were in line with the antigenic fingerprints of the DT-IPV vaccines. Except for PadPO, the luminescence intensities were on average 1.2-3.5 times higher for all mabs than the responses in the DT-IPV vaccines (Figs. $2 \mathrm{~A}$ and $3 \mathrm{~A}$ ). The higher DTd concentrations of adDTd-X and adDTd-Y (15 Lf/ml) as compared to the DT-IPV vaccines $(2.5 \mathrm{Lf} / \mathrm{ml})$ might be the reason that the luminescence intensities were higher.

\subsection{Antigenic fingerprints of adsorbed and pre-adsorbed diphtheria toxin}

Diphtheria toxin (DTx) is more prone to conformational changes upon adsorption than DTd, due to the lack of stabilising covalent cross-links induced during inactivation. Therefore, DTx was also included in this study.

The changes in the antigenic fingerprint of adsorbed DTx were significantly different compared the pre-adsorbed and desorbed state of DTx (Fig. 3A and B). The epitopes of Dim27 and Dim25 disappeared almost completely in DTx after adsorption. Moreover, the epitopes of Dim9, Dim24 and DiD1 became less accessible compared to DTd-X and DTd-Y. Dim9 and Dim24 bound with higher intensities to pre-adsorbed or desorbed DTx than to the preadsorbed and desorbed DTd, whereas DiD1 bound with equal intensity (Fig. 3A and B).

In pre-adsorbed state, the binding intensities of Dim9, Dim24, Dim27 and DiD1 to DTx were not significantly different from each other. However, the responses of Dim25 and PaDPO were significantly lower (Fig. 3B). Furthermore, the desorbed DTx concentrations were approximately $55-75 \%$ of the pre-adsorbed DTx concentrations. Incomplete recovery of DTx suggest that 

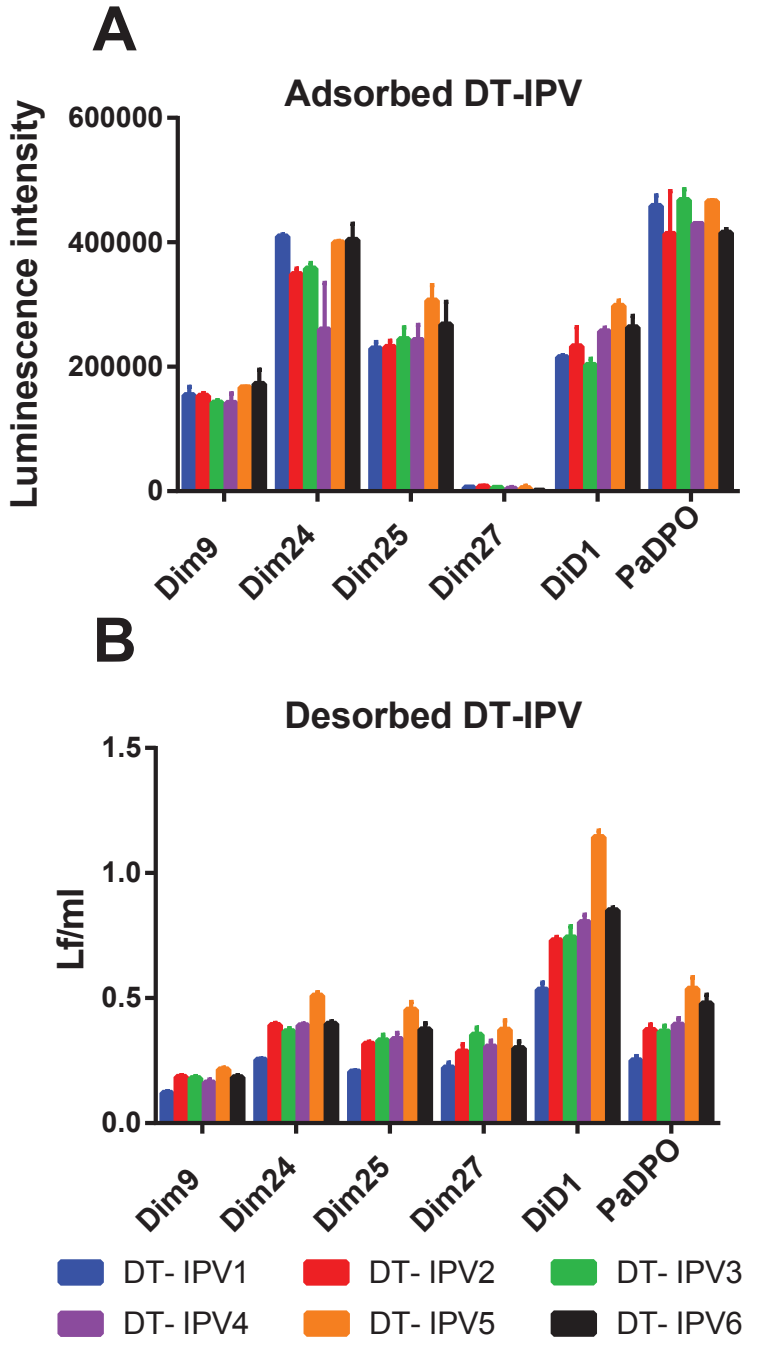

Fig. 2. Antigenic fingerprints of aluminium phosphate-adsorbed (A) and desorbed (B) DT-IPV-vaccines DT-IPV1 (blue), DT-IPV2 (red), DT-IPV3 (green), DT-IPV4 (purple), DTIPV5 (orange) and DT-IPV6 (black). Panel A shows the DTd epitope response to the mabs or PaDPO, expressed as the mean luminescence intensity at $0.08 \mathrm{Lf} / \mathrm{ml}$ DT-IPV after subtraction of the luminescence intensity of the negative control (plain aluminium phosphate at equal concentration, i.e. $10 \mathrm{ng} / \mathrm{ml} \mathrm{Al}^{3+}$ ). Error bars depict SD at $\mathrm{n}=2$. Panel $\mathrm{B}$ shows the mean DTd concentration in $\mathrm{Lf} / \mathrm{ml}$ in desorbed DT-IPV vaccines. Error bars depict SD at $n \geq 6$, except for $\operatorname{Dim} 25$ ( $n \geq 3$ ). (For interpretation of the references to colour in this figure legend, the reader is referred to the web version of this article.)

desorption of DTx was not complete or the desorption process degraded the toxin to some extent. DTx is less stable than DTd, because formaldehyde-induced cross-links are not present in DTx. In conclusion, the adsorption of DTx resulted in a distinct antigenic fingerprint which is mostly reversible after desorption.

\section{Discussion}

Aluminium-containing vaccines are complicated products and difficult to analyse with the classical protein characterization techniques. The lack of in vitro assays for final adjuvated products is one of the reasons why potency testing of toxoid-containing vaccines is not yet replaced by in vitro tests. The use of physicochemical techniques is helpful to analyse monovalent adsorbed vaccines. However, these analytical techniques have a major drawback, i.e. they cannot be used in combination vaccines because of lack of
A

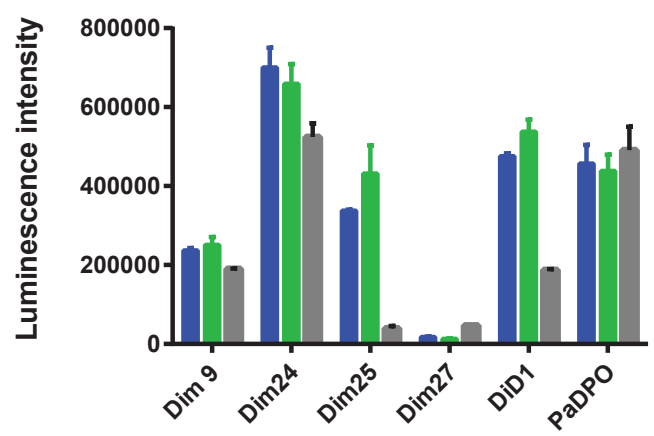

B

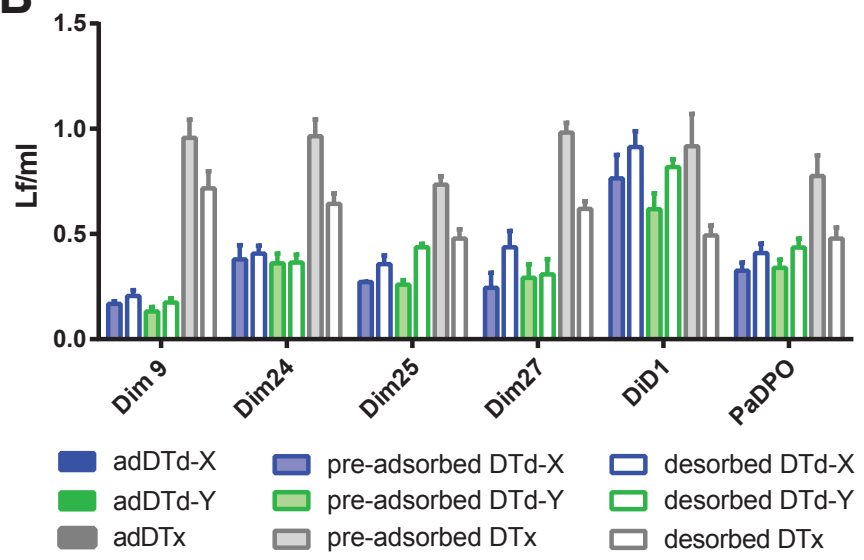

Fig. 3. Antigenic fingerprints of aluminium phosphate-adsorbed (A), pre-adsorbed and desorbed (B) DTd-X (blue), DTd-Y (green) and DTx (grey) vaccines. Panel A shows the DTd/DTx epitope response to the mabs or PaDPO, expressed as the mean luminescence intensity at $0.5 \mathrm{Lf} / \mathrm{ml}$ adDTd or adDTx after subtraction of the luminescence intensity of the negative control (plain aluminium phosphate at $10 \mathrm{ng} / \mathrm{ml} \mathrm{Al}^{3+}$ ). Error bars depict $\mathrm{SD}$ at $\mathrm{n}=2$. Panel B shows the mean DTd/DTx concentration in $\mathrm{Lf} / \mathrm{ml}$ in pre-adsorbed (filled bar) and desorbed (empty bar) DTd-X (blue), DTd-Y (green) and DTx (grey). Error bars depict SD at $n \geq 6$, except for $\operatorname{Dim} 25$ ( $n \geq 3$ ). (For interpretation of the references to colour in this figure legend, the reader is referred to the web version of this article.)

specificity. The use of immunoassays on adsorbed antigens solves some of these problems and may give valuable information about product consistency between vaccine lots.

To demonstrate comparability of alum adsorbed combination vaccines relevant product parameters have to be identified and analysed. One of them, the degree of adsorption is already determined routinely and manufacturers have set specifications for degree of adsorption. It may be that in addition to or instead of adsorption degree the affinity of antigens for alum adjuvant is important [7]. An indication of adsorption strength can be obtained by determining elutability in buffer mimicking interstitial fluid. A second parameter is the quality of the alum adjuvant. Particle size and shape, aggregation state and surface charge may influence interaction with the antigen as well as the intrinsic immune modulatory effects of aluminium salts. A third parameter, addressed here in this paper, is the antigenic quality of the adsorbed proteins.

For an antigenic fingerprint it is important to use mabs that recognize different epitopes and preferably relevant epitopes, i.e. toxin neutralising. But to demonstrate product consistency one could also focus on epitopes that are sensitive to conformational changes. The monoclonals used in this study were extensively characterised (specified in Materials and Methods). 
The sensitivity of the in vitro assay for adsorbed DTd is theoretically high enough to be useful in future batch release. Antigenic fingerprints could be obtained from all six DT-IPVs which met the release criteria. Relatively small but significant differences were shown between the six DT-IPV vaccines in the responses toward Dim24 (DT-IPV4 had the lowest response), Dim25 and DiD1 (DTIPV5 had the highest response to both mabs) (Fig. 2A). Before batch release can be done with the in vitro assay, specifications and alarm limits have to be defined, i.e. by side-by-side testing of the vaccines in regular animal tests and the new assay.

The experimental vaccines, adDTd-X and adDTd-Y contain six times more DTd than the DT-IPV vaccines at a constant aluminium concentration. The DTd/DTx concentration was deliberately chosen because most of the commercially available DTd-containing vaccines have a concentration of $15 \mathrm{Lf} / \mathrm{ml}$. The $2.5 \mathrm{Lf} / \mathrm{ml}$ DTdcontaining DT-IPV vaccines are an exception and used as a traveller vaccine. The luminescence intensities of the adDTd-X and adDTd-Y were on average 1.2-3.5 times higher for all mabs than the responses in the DT-IPV vaccines (Figs. $2 \mathrm{~A}$ and $3 \mathrm{~A}$ ). To maintain a constant aluminium concentration, the read out of the luminescence intensity for DT-IPV vaccines and experimental adDTd vaccines was 64 times dilution in a twofold dilution series meaning that the 64 times dilution of the experimental DTd vaccines contained also a six time higher DTd concentration than the DT-IPV vaccines. The reason that the luminescence intensities did not correlate with the differences in DTd concentration may be caused by the aluminium phosphate used and the adsorption procedure. The experimental DTd products were adsorbed to aluminium phosphate from Brenntag, whereas the DT-IPV vaccines were adsorbed to aluminium phosphate prepared in situ. Shielding of epitopes to a greater or lesser extent by aluminum phosphate might be a reason for a difference in luminescence intensity. This also emphasizes that, as mentioned above, characterization of the alum adjuvants themselves may be important to demonstrate consistency of vaccines containing these adjuvants.

Chang et al. demonstrated that antigen might desorb after in vivo application [7]. Also, for B-cell recognition it is probably necessary that the antigen desorbs at some point after administration. Therefore, we investigated whether the changes in the epitope fingerprint were reversible after in vitro desorption. After desorption, differences in epitope binding between the six DT-IPV batches became more prominent. From the six DT-IPVs, DT-IPV5 showed the highest antigenicity. Speculatively, this may be caused by differences in aluminum phosphate quality. Every final DT-IPV contained a unique aluminum phosphate batch. If aluminum phosphate in DT-IPV1 has a higher affinity for DTd than DT-IPV5, the adsorption may change the conformation of DTd more than it did in DT-IPV5. This might result in lower antigenicity in the adsorbed state. If stronger bound DTd is refolding to the native state after desorption, the folding process might be slower or incomplete. This results in a relatively low ELISA signal. Adsorbed DTd in DT-IPV5 is probably more loosely bound and less unfolded. As a result DTd refolds completely after desorption, resulting in a higher ELISA signal.

Regarding the measured DTd concentration of the pre-adsorbed and desorbed DT-IPV vaccines, one should expect that this concentration would be around the $1.2 \mathrm{Lf} / \mathrm{ml}$, which is the starting concentration of the diluted DT-IPV vaccines and reference DT79/1. Except for DiD1, the calculated epitope concentrations were all between 0.14 and $0.65 \mathrm{Lf} / \mathrm{ml}$ (Figs. 2B and 3B). This may be caused by the fact that the reference DT79/1 is a DTx, whereas DT-IPV vaccines contain of course DTd. The availability of A- and B-fragment epitopes of DTx is higher as compared to the epitopes of DTd caused by formaldehyde treatment of DTx [22]. The DTd concentrations of desorbed experimental DTd-X and DTd-Y were slightly higher than the DTd concentrations in pre-adsorbed state. It could be that subsequent steps of adsorption and desorption dissolves small DTd aggregates resulting in higher antigen values $(\mathrm{Lf} / \mathrm{ml})$.

In adsorbed state no binding of Dim27 was observed in DT-IPVs, adDTd-X and adDTd-Y (Figs. 2A and 3A). However, binding of DTd to Dim27 was evident after desorption (Figs. 2B and 3B). Changes in epitope profile observed during adsorption of DTd disappeared after desorption. These changes could be caused by a certain preference of the antigen to adsorb to aluminium phosphate by which certain epitopes disappear. The steric hindrance of the epitopes by aluminium phosphate result in reduced antibody binding to adsorbed DTd. Previously, we demonstrated that DTd undergoes substantial conformational changes upon adsorption to aluminium hydroxide by using spectroscopic techniques, i.e. fluorescence and circular dichroism, at least to aluminium hydroxide [17]. After desorption, DTd started to refold to its original conformation. The present study showed that these conformational changes are reversible after desorption.

Changes in epitope profile were even more significant when diphtheria toxin was adsorbed instead of toxoid. In the preadsorbed situation, the binding intensity of the mabs to DTx was similar, except for Dim25 and PadPO. The binding of these mabs had a significantly lower intensity. However, in the adsorbed state epitopes of Dim24 and PaDPO had a higher luminescence intensity and the Dim25 and Dim27 epitopes became almost undetectable as a result of the adsorption. After desorption the antigenic fingerprint had a similar profile as compared to the pre-adsorbed situation, although fluorescence intensities of desorbed DTx were only 55-75\% compared pre-adsorbed DTx. The difference in adsorption/ desorption behaviour between DTd and DTx is probably due to the formaldehyde treatment of DTx. Inactivation results in the formation of intra-molecular cross-links and strongly increased conformational stability [22].

The current paper describes the use of an adapted version of an aluminium-adsorbed antigen immunoassay to obtain antigenic fingerprints of adsorbed antigens present in vaccines. Antigenic fingerprinting has potential to be used for consistency testing of adsorbed combination vaccines in an antigen specific way, without the need to desorb or otherwise pretreat the final vaccine. In addition, the antigenic fingerprints demonstrate that the availability of epitopes can change significantly, but they become accessible again after desorption.

Finally, we recommend an investigation into the feasibility of the aluminium-adsorbed antigen immunoassay for other aluminium-containing adjuvants, e.g. aluminium hydroxide. Furthermore, extensive validation studies are needed to confirm the reliability, reproducibility and robustness of the test for quality control.

\section{Acknowledgements}

The study was part of a project on 3Rs alternatives to animal use in vaccine quality control and is funded by the Dutch Ministry of Health (Project number AV/900096), Welfare and Sports and the Dutch Ministry of Economic Affairs'.

\section{References}

[1] Kool M, Fierens K, Lambrecht BN. Alum adjuvant: some of the tricks of the oldest adjuvant. J Med Microbiol 2012;61:927-34.

[2] Marichal T, Ohata K, Bedoret D, Mesnil C, Sabatel C, Kobiyama K, et al. DNA released from dying host cells mediates aluminum adjuvant activity. Nat Med 2011;17:996-1002.

[3] Glenny AT, Buttle GAH, Stevens MF. Rate of disappearance of diphtheria toxoid injected into rabbits and Guinea pigs: toxoid precipitated with Alum. J Pathol Bacteriol 1931;34:267-75.

[4] MacLean DS, Robertson JD, Jay M. Monitoring the retention of a protein 
antigen in complete Freund's adjuvant, alum, and pluronic F-127 gel formulations by X-ray fluorescence. Pharm Dev Technol 2001;6:241-6.

[5] Ghimire TR. The mechanisms of action of vaccines containing aluminum adjuvants: an in vitro vs in vivo paradigm. Springerplus 2015;4:181.

[6] Morefield GL, Sokolovska A, Jiang D, HogenEsch H, Robinson JP, Hem SL. Role of aluminum-containing adjuvants in antigen internalization by dendritic cells in vitro. Vaccine 2005;23:1588-95.

[7] Chang M, Shi Y, Nail SL, HogenEsch H, Adams SB, White JL, et al. Degree of antigen adsorption in the vaccine or interstitial fluid and its effect on the antibody response in rabbits. Vaccine 2001;19:2884-9.

[8] Egan PM, Belfast MT, Gimenez JA, Sitrin RD, Mancinelli RJ. Relationship between tightness of binding and immunogenicity in an aluminum-containing adjuvant-adsorbed hepatitis B vaccine. Vaccine 2009;27:3175-80.

[9] Hansen B, Sokolovska A, HogenEsch H, Hem SL. Relationship between the strength of antigen adsorption to an aluminum-containing adjuvant and the immune response. Vaccine 2007;25:6618-24.

[10] Berthold I, Pombo ML, Wagner L, Arciniega JL. Immunogenicity in mice of anthrax recombinant protective antigen in the presence of aluminum adjuvants. Vaccine 2005;23:1993-9.

[11] Romero Mendez IZ, Shi Y, HogenEsch H, Hem SL. Potentiation of the immune response to non-adsorbed antigens by aluminum-containing adjuvants. Vaccine 2007;25:825-33.

[12] Hansen B, Belfast M, Soung G, Song L, Egan PM, Capen R, et al. Effect of the strength of adsorption of hepatitis B surface antigen to aluminum hydroxide adjuvant on the immune response. Vaccine 2009;27:888-92.

[13] EMA. Guideline on adjuvants in vaccines for human use. 2005.
[14] Iyer S, HogenEsch H, Hem SL. Effect of the degree of phosphate substitution in aluminum hydroxide adjuvant on the adsorption of phosphorylated proteins. Pharm Dev Technol 2003;8:81-6.

[15] Seeber SJ, White JL, Hem SL. Solubilization of aluminum-containing adjuvants by constituents of interstitial fluid. JParenterSciTechnol 1991;45:156-9.

[16] Clapp T, Siebert P, Chen D, Jones Braun L. Vaccines with aluminum-containing adjuvants: optimizing vaccine efficacy and thermal stability. J Pharm Sci 2011;100:388-401.

[17] Regnier M, Metz B, Tilstra W, Hendriksen C, Jiskoot W, Norde W, et al Structural perturbation of diphtheria toxoid upon adsorption to aluminium hydroxide adjuvant. Vaccine 2012;30:6783-8.

[18] Zhu D, Huang S, Gebregeorgis E, McClellan H, Dai W, Miller L, et al. Development of a direct Alhydrogel formulation immunoassay (DAFIA). J Immunol methods 2009;344:73-8.

[19] Ugozzoli M, Laera D, Nuti S, Skibinski DA, Bufali S, Sammicheli C, et al. Flow cytometry: an alternative method for direct quantification of antigens adsorbed to aluminum hydroxide adjuvant. Anal Biochem 2011;418:224-30.

[20] Zhu D, Saul A, Huang S, Martin LB, Miller LH, Rausch KM. Use of o-phthalaldehyde assay to determine protein contents of Alhydrogel-based vaccines. Vaccine 2009;27:6054-9.

[21] Metz B. Structural characterisation of diphtheria toxoid. Utrecht: Utrecht University; 2005.

[22] Metz B, Jiskoot W, Hennink WE, Crommelin DJA, Kersten GFA. Physicochemical and immunochemical techniques predict the quality of diphtheria toxoid vaccines. Vaccine 2003;22:156-67. 